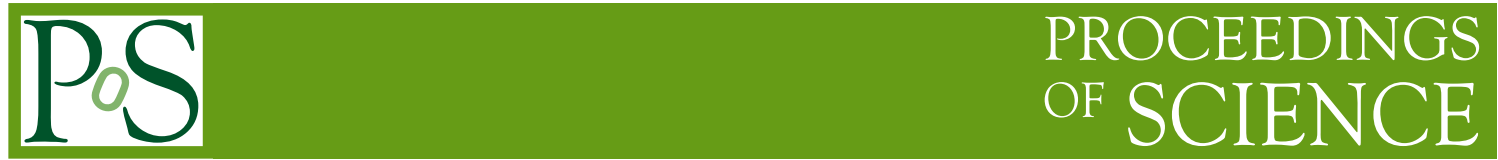

\title{
Relativistic superluminal radio jets in microquasars in our galaxy
}

\author{
J S Yadav* \\ TATA Institute of Fundamental Research, Mumbai, India \\ E-mail: jsyadav@crspa.tifr.res.in
}

\begin{abstract}
We discuss the origin of superluminal radio jets in Black hole X-ray binaries with relativistic radio jets in our Galaxy popularly known as microquasars. We classify the relativistic superluminal jet according to the radio emission in black hole X-ray binaries (transient or persistent) rather than the mass of the companion. The black hole $\mathrm{X}$-ray binaries with transient radio emission (mostly LMXBs) produce superluminal jets with $\beta_{a p p}>1$ when the accretion rate, $\dot{m}_{a c c r}$ is high and the bolometric luminosity $\mathrm{L}_{b o l}$ approaches the Eddington Luminosity, $\mathrm{L}_{E d d}$. On the other hand, the black hole X-ray binaries with persistent radio emission (mostly HMXBs) produce superluminal jets with $\beta_{a p p}<1$ at relatively low $\dot{m}_{a c c r}$. We specially discuss the case of V4641 Sgr, a HMXB with transient radio emission which produces superluminal radio jets like in LMXBs.
\end{abstract}

VI Microquasar Workshop: Microquasars and Beyond

September 18-22, 2006

Como, Italy

\footnotetext{
* Speaker.
} 


\section{Introduction}

The Black hole X-ray binaries with relativistic radio jets have earned the name "microquasars" as they are stellar-mass analogs of the massive extragalactic black hole systems in Quasars and Active Galactic Nuclei (AGNs) [1,2]. The total number of X-ray binaries (HMXBs plus LMXBs) as per the recent catalogues is 280 which includes neutron star and black hole X-ray binaries [3, 4]. Most of the LMXBs are transients while most of the HMXBs are persistent. The current number of microquasars is $\sim 14$ which are listed in Table 1 out of total $43 \mathrm{X}$-ray binaries in which radio emission is detected $[5,6]$.

Mirabel \& Rodriguez (1994) discovered first microquasar GRS 1915+105 in our galaxy with superluminal jets. This microquasar shows exceptionally high variability in both $\mathrm{X}$-rays and radio $[7,8,9]$. All the radio flares observed in GRS $1915+105$ can be broadly put into two groups on the basis of their flux, radio spectrum and spatial distribution; (1) the superluminal flares (200-1000 mJy) which have steep radio spectra and are seen at large distances ( $\geq 240 \mathrm{AU})$, and (2) all other flares (5-360 mJy) which include the preplateau flares, radio oscillations \& discrete flares and the steady radio flares during the plateaux. All these flares have flat or inverted radio spectra and are observed close to the compact object $(<200 \mathrm{AU})$. These radio flares can be understood as mass ejections of adiabatically expanding self absorbing synchrotron clouds [10, 11, 12, 13].

The physical connection between X-ray emission and the superluminal flares has been the hardest to understand[14, 15]. Recently, Yadav (2006) has studied X-ray properties during plateaux and the following superluminal radio jets in GRS 1915+105 and has provided tight correlation between the accretion disk and the radio jet parameters. It is suggested that the superluminal jets are due to the internal shock which forms when the oscillation or discrete baby jet (compact jet) catches up and interact with the previously generated slowly moving wind from the accretion disk (see next section for details). We attempt in this paper to broaden this understanding of the superluminal radio jets to HMXBs with mostly persistent radio emission in which the wind from the companion is the usual source of accretion. Cyg X-3 is the most active in this class of HMXBs. This source

\begin{tabular}{|lccccc|}
\hline \hline Source & $\begin{array}{c}\text { Type of } \\
\text { binary }\end{array}$ & $\begin{array}{c}\mathrm{D} \\
\mathrm{kpc})\end{array}$ & $\begin{array}{c}\mathrm{M}_{\text {compact }} \\
\mathrm{M}_{\odot}\end{array}$ & $\begin{array}{c}\text { Radio } \\
\text { emission }\end{array}$ & $\beta_{\text {appr }}$ \\
\hline LS I +61 303 & HMXB & 2.0 & - & $\mathrm{p}$ & $\geq 0.4$ \\
V4641 Sgr & HMXB & $\sim 10$ & 9.6 & $\mathrm{t}$ & $\geq 9.5$ \\
LS 5039 & HMXB & 2.9 & $3(?)$ & $\mathrm{p}$ & $\geq 0.15$ \\
SS 433 & HMXB & 4.8 & $11 \pm 5(?)$ & $\mathrm{p}$ & 0.26 \\
Cygnus X-1 & HMXB & 2.5 & 10.1 & $\mathrm{p}$ & - \\
Cygnus X-3 & HMXB & 9 & $-(?)$ & $\mathrm{p}$ & 0.69 \\
XTE 1118+480 & LMXB & 1.9 & $6.9 \pm 0.9$ & $\mathrm{t}$ & - \\
XTE J1550-564 & LMXB & 5.3 & 9.4 & $\mathrm{t}$ & $>2$ \\
GRO J1655-40 & LMXB & 3.2 & 7.02 & $\mathrm{t}$ & 1.1 \\
GX 339-4 & LMXB & $>6$ & $5.8 \pm 0.5$ & $\mathrm{t}$ & $\geq 2.2$ \\
1E 1740.7-2942 & LMXB & $8.5(?)$ & - & $\mathrm{p}$ & - \\
XTE J1748-288 & LMXB & $\geq 8$ & $>4.5(?)$ & $\mathrm{t}$ & 1.3 \\
GRS 1758-258 & LMXB & $8.5(?)$ & - & $\mathrm{p}$ & - \\
GRS 1915+105 & LMXB & 12.5 & $14 \pm 4$ & $\mathrm{t}$ & $1.2-1.7$ \\
\hline
\end{tabular}

Table 1: Microquasars in our Galaxy. Radio jet sources with neutron stars like Circinus X-1 and Scorpius X1 are not included. 


\begin{tabular}{|llll|llll|}
\hline \hline \multicolumn{4}{|c|}{ Radio Flare Properties } & \multicolumn{4}{c|}{ Associated X-ray properties } \\
\hline MJD & Source & $\begin{array}{l}\text { Type of } \\
\text { flare }\end{array}$ & $\begin{array}{l}\text { Flux(MJy) } \\
2.25 \mathrm{GHz}\end{array}$ & MJD/Day & $\begin{array}{l}\text { X-ray } \\
\text { Flux }\end{array}$ & $\begin{array}{l}\mathrm{N}_{H} \\
\left(10^{22} \mathrm{~cm}^{-2}\right)\end{array}$ & $\begin{array}{l}\chi_{v}^{2}(\mathrm{dof}) \\
(\text { dof })\end{array}$ \\
\hline 50915 & GRS 1915+105 & Superluminal & 920 & 50913/1998 Apr. 11 & 2.14 & $14.95_{-0.65}^{+0.82}$ & $0.83(94)$ \\
52105 & GRS 1915+105 & Superluminal & 368 & 52101/2001 Jul. 11 & 1.61 & $10.60_{-0.40}^{+0.77}$ & $0.98(87)$ \\
50674 & GRS 1915+105 & Oscil./baby jets $^{b}$ & $60-80^{c}$ & 50674/1997 Aug. 14 & 1.00 & $7.0^{d}$ & $0.92(75)$ \\
50700 & GRS 1915+105 & Oscil./baby jets & $60-80^{c}$ & 50700/ 1997 Sep. 9 & 1.01 & $7.0^{d}$ & $0.71(75)$ \\
50610 & Cyg X-3 & Superluminal & 4000 & 50605/ 1997 Jun. 5 & 0.76 & $28.91_{-0.40}^{+1.50}$ & $0.99(70)$ \\
51654 & Cyg X-3 & Superluminal & 13000 & 51646/ 2000 Apr. 12 & $0.45^{e}$ & $25.53_{-7.00}^{+14.00}$ & $0.83(64)$ \\
\hline
\end{tabular}

${ }^{a}$ Integrated 4-150 keV X-ray flux in $10^{-8} \mathrm{ergs} \mathrm{cm}^{-2} \mathrm{~s}^{-1}$

${ }^{b}$ Radio flux is calculated from IR flux after dereddening, ${ }^{c}$ Radio flux at $8.3 \mathrm{GHz}$

${ }^{d}$ This value is for fixed $\mathrm{N}_{H}$. This is also consistent with variable $\mathrm{N}_{H}$ which results $8.40_{-1.45}^{+1.50}$

${ }^{e}$ Only 20 min RXTE PPC/HEXTE data and both the total X-ray flux and $\mathrm{N}_{H}$ are likely to be underestimated

Table 2: All selected superluminal radio jets, oscillation/baby jets and their X-ray Properties from RXTE PCA/HEXTE data

was first detected in 1967 [16], and is known to produce huge superluminal radio jets [17, 18]. We have studied X-ray properties during the plateaux \& the class $\beta$ in GRS $1915+105$ and during the low hard state in Cyg X-3 preceding superluminal flares. We also studied radio properties of superluminal and discrete/oscillation jets (see Table 2). For X-ray spectral analysis, we have used REXTE PCA/HEXTE data in the range $4-150 \mathrm{keV}$. The decay time constant of a superluminal flare is calculated by fitting exponential decay profile to the $2.25 \mathrm{GHz}$ GBI radio monitoring data. Yadav (2006) has given details of X-ray and radio data analysis.

\section{Results and discussion}

We list in Table 1 data of all the known microquasars. We have limited our list to only black hole X-ray binaries. Most of the HMXBs in Table 1 show persistent radio emission while most of the LMXBs are transient in nature. It is clear from Table 1 that all the microquasars with transient radio emission produce superluminal jets with $\beta_{\text {appr }}>1$ while those with persistent radio emission produce superluminal jets with $\beta_{\text {appr }}<1$.

Yadav (2006) has shown that the accretion rate, $\dot{m}_{a c c r}$ is very high during the radio plateaux in GRS $1915+105$ and the bolometric luminosity $\mathrm{L}_{b o l}$ approaches Eddington Luminosity $\mathrm{L}_{E d d}$. It is suggested that such luminous accretion disk during the plateaux always accompany with radiationdriven wind. The internal shock forms in the previously generated slowly moving wind from the accretion disk during the plateaux with $\beta \leq 0.01$ as the fast moving discrete jet with $\beta \sim$ 1 catches up and interacts with it $[19,20]$. Both the components; slow moving wind and fast moving jet are related to the accretion disk during plateau state and the strength \& speed of these two should determine the power of the internal shock and of the superluminal radio jet. A tight correlation is observed between peak flux of superluminal flares and absorption column density, $\mathrm{N}_{H}$ with correlation coefficient of 0.99 (see our other paper in this proceeding) which suggests that for wind strength corresponding to $\mathrm{N}_{H} \leq 8.3 \pm 1.5 \times 10^{22} \mathrm{~cm}^{-2}$, no superluminal jet will be produced. The superluminal radio jets in most of LMXBs are produced when the source is in very high luminous state (VHS) like plateaux in GRS 1915+105 a prerequisite to produce slow moving strong wind. The evidences for a Seyfert-like warm absorber have been found in many LMXBs 


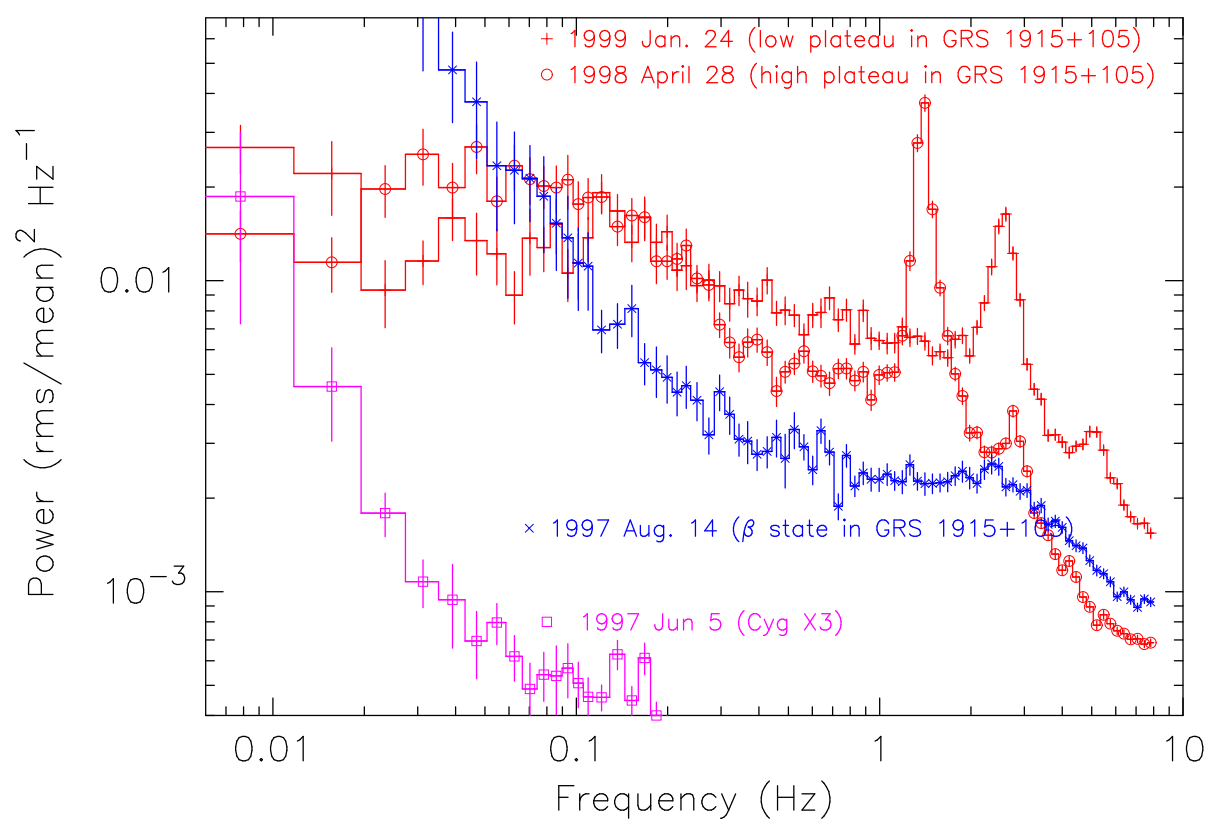

Figure 1: Normalised power density spectra in $0.007-9 \mathrm{~Hz}$ frequency range observed during the plateaux state (1998 April 28 and 1999 January 24) and during the X-ray class $\beta$ on 1997 August 14 in GRS 1915+105. The PDS observed during the low hard state on 1997 June 5 in Cyg X-3 preceding superluminal flare.

like GRO J1655-40, GX 339-4 and XTE J1650-500 beside GRS 1915+105 [21, 22, 23]. The $\beta_{\text {appr }}$ of observed superluminal jets in these systems is $>1$ and peak radio flux is in the range of $0.4-2$ Jy. The rise time of superluminal flares in these sources is in the range $0.25-0.5$ day $[19,15]$.

The absence of superluminal jets during the class $\beta$ in GRS $1915+105$ is attributed to the absence of wind [19]. In Table 2, we give details of radio flares and the associated X-ray properties during the plateaux and the class $\beta$ in GRS $1915+105$. During the X-ray class $\beta$ in GRS $1915+105$, the total X-ray flux is almost half than that observed during the plateaux and the calculated $\mathrm{N}_{H}$ is below the critical $\mathrm{N}_{H}$ discussed above to produced superluminal jets. We also present in Table 2 the radio properties of two superluminal flares observed in Cyg X-3 along with associated X-ray properties during the preceding low hard state. The $\mathrm{N}_{H}$ is in the range of $25-$ $30 \times 10^{22} \mathrm{~cm}^{-2}$ which is consistent with the strong wind from the companion. The total X-ray flux is close to that observed during the class $\beta$ in GRS $1915+105$. The observed $\mathrm{N}_{H}$ and the X-ray flux in Cyg X-3 will suggest that the discrete/oscillation compact jet may interact with the wind which originates from the companion to produces superluminal jets in HMXBs with persistent radio emission.

Figure 1 shows PDS spectra observed during low plateau on 1999 January 24 and during high plateau on 1998 April 28 in GRS 1915+105 . Yadav (2006) has suggested that the fast variability is suppressed by photon scattering in the enhanced wind on 1998 April 28 and hence reducing the power in the PDS at higher frequencies (see Paper 1 for detailed discussion). We also show in Figure 1 the calculated PDS for class $\beta$ in GRS 1915+105 and during low hard state in Cyg X-3 . The shape of PDS during class $\beta$ in GRS $1915+105$ is similar to the shape of PDS observed in 


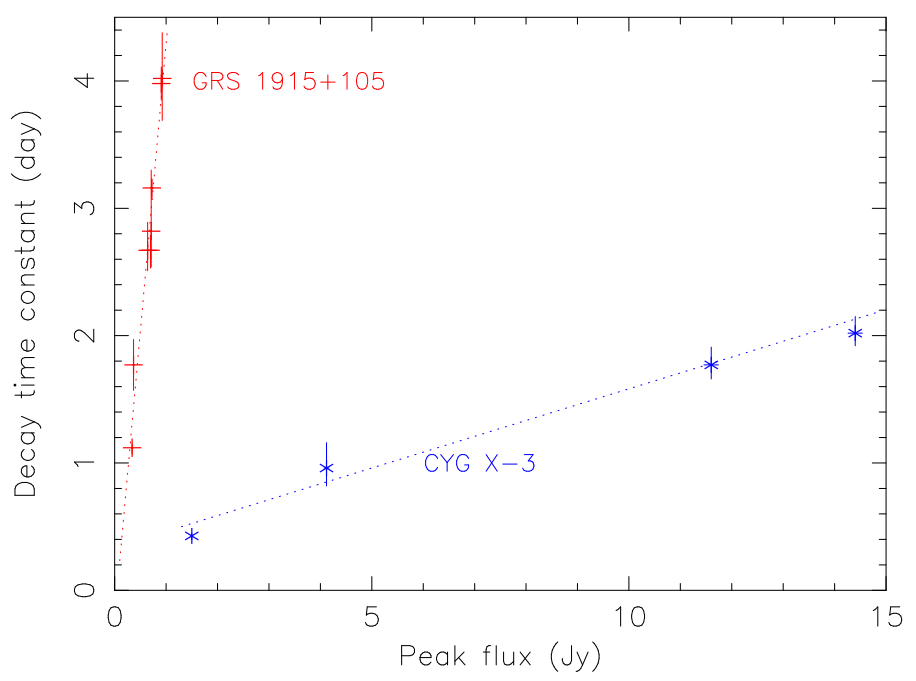

Figure 2: The peak flux of the superluminal flares as a function of decay time constant of the superluminal flares observed in GRS 1915+105 and Cyg X-3 from the analysis of $2.25 \mathrm{GHz}$ GBI data.

Cyg X-3 except the PDS power in Cyg X-3 at higher frequency is highly suppressed due to dense wind from the companion [24]. The depleted IR emission in Cyg X-3 as compared to class $\beta$ in GRS 1915+105 also indicates the presence of strong wind in Cyg X-3 [19].

In the case of HMXBs with persistent radio emission like Cyg X-3, superluminal jets are produced at relatively low $\dot{m}_{a c c r}$ as the slow moving medium; wind is provided by the companion. In this case power of superluminal jets is coming from the accretion disk as well as from the wind which originates from the companion. The superluminal flare (transient) mode boosts the radio luminosity over the $\mathrm{X}$-ray luminosity in microquasars and the boosting of radio luminosity is higher in Cyg X-3 than that in GRS 1915+105 [25]. This is because the wind from the companion provide additional power to superluminal jets in Cyg X-3. Figure 2 shows decay time constant as a function of peak flux of superluminal flares observed in GRS 1915+105 and Cyg X-3 obtained from the analysis of $2.25 \mathrm{GHz}$ GBI monitoring data. The slow wind and the compact jet both originate from the accretion disk in GRS 1915+105 while the wind from the companion provides accreting material as well as slow moving medium to evolve internal shock in Cyg X-3 and hence both follow linear rise. The GRS $1915+105$ data represent superluminal jets produced when $\dot{m}_{a c c r}$ is very high and the $\mathrm{L}_{b o l}$ approaches $\mathrm{L}_{E d d}$ while Cyg X-3 data represent superluminal jets produced at relatively low $\dot{m}_{a c c r}$ but with dense wind from the companion. Superluminal jets from other microquasars should fall between these two.

The case of V4641 Sgr is special as it is a HMXB with transient radio emission and has produced superluminal jets with $\beta_{a p p r} \geq 9.5$ (Table 1) [26]. The giant outburst in 1999 September 14-15 is attributed to an episode of super-Eddington accretion onto the black hole and the $\mathrm{L}_{b o l}$ approaches $\mathrm{L}_{E d d}$ [27]. During this outburst, extended optically thick envelope/outflow has been seen. Optical outbursts have been seen earlier in GRO J1655-40 and XTE J1550-564, both of them LMXBs with transient radio emission. During the outburst, wind is reported and the $\mathrm{N}_{H}$ has increased substantially which is similar to what we observe during the plateaux in GRS 1915+105 [27, 19]. However these plateau like conditions lasted for short duration less than a day (1999 Septem- 
ber 14.9-15.7). The elongated radio jet with steep radio spectra was seen on September 16 with peak flux $\sim 400$ mJy which disappeared quickly [26]. This would suggest a partially developed internal shock due to lack of slow moving medium; the wind. It suggests that V4641 Sgr behaves like LMXBs with transient radio emission.

\section{Acknowledgments}

The author thanks RXTE PCA/HEXTE and NSF-NRAO-NASA Green Bank Interferometer teams for making their data publicly available.

\section{References}

[1] Mirabel, I. F., \& Rodriguez, L. F. 1994, Nature, 371, 46

[2] Tingay, S. et al. 1995, Nature, 374, 141

[3] Liu, O. Z., van Paradijs, J. \& van den Heuvel, E.P.J.,2000, A\&AS, 147, 25

[4] Liu, O. Z., van Paradijs, J. \& van den Heuvel, E.P.J., 2001, A\&A, 368, 1021

[5] McClintock, J. E. \& Remillard, R. A. 2004, Astro-ph/0306213 v3

[6] Paredes, J. M. 2005, Astro-ph/0501576 v1

[7] Muno, M. P., Morgan, E. H. \& Remillard, R. A. 1999, ApJ, 527, 321

[8] Yadav, J. S., Rao, A. R., Agrawal, P. C, et al. 1999, ApJ, 517, 935

[9] Belloni, T., Klein-Wolt, M., Mendez, M., et al. 2000, A\&A, 355, 271

[10] Mirabel, I. F., Dhawan, V., Chaty, S. et al. 1998, A\&A, 330, L9

[11] Eikenberry, S. S., Matthews, K., Morgan, E. H., et al. 1998, ApJ, 494, L61

[12] Yadav, J. S. 2001, ApJ, 548, 876

[13] Ishwara-Chandra, C. H., Yadav, J. S., \& Pramesh Rao, A. 2002, A\&A, 388, L33

[14] Fender, R. P. \& Belloni, T. M. 2004, ARA\&A, 42, 317

[15] Fender, R. P., Belloni, T. M. \& Gallo, E. 2004, MNRAS, 355, 1105

[16] Giacconi, R. et al. 1967, ApJ, 148, L119

[17] Miller-Jones, J. C. A. et al. 2004, ApJ, 600, 368

[18] Mioduszewski, A. J. et al. 2001, ApJ, 553, 766

[19] Yadav, J. S., 2006, ApJ, 646, 385

[20] Kaiser, C. R., Sunyaev, R. \& Spruit, H. C. 2000, A\&A, 356, 975

[21] Miller, J. M., Raymond, J., Fabian, A. C., et al. 2004, ApJ, 601, 450

[22] Ueda, Y., Inoue, H., Tanaka, Y., Ebisawa, K., et al. 1998, ApJ, 492, 782

[23] Kotani, T., Ebisawa, K., Dotani, T., et al. 2000, ApJ, 539, 413

[24] Choudhury, M., Rao, A. R., Vadawale, S. V. et al. 2004, A\&A, 420, 665

[25] Nipoti, C., Blundell, K. M. \& Binney, J. 2005, MNRAS, 361, 633

[26] Hjellming, R. M., \& Rupen, M. P., Hunstead, D. et al.,2000, ApJ, 544, 977

[27] Revnivtsev, M. et al. 2002, A\&A, 391, 1013 